\title{
Correction to: Cognitive profiles discriminate between genetic variants of behavioral frontotemporal dementia
}

\author{
J. M. Poos ${ }^{1,2}$ (1) $\cdot$ L. C. Jiskoot ${ }^{1,3} \cdot$ S. M. J. Leijdesdorff ${ }^{4}$ H. Seelaar ${ }^{1}$. J. L. Panman ${ }^{1,2} \cdot$ E. L. van der Ende ${ }^{1} \cdot$ M. O. Mol ${ }^{1}$. \\ L. H. H. Meeter ${ }^{1}$. Y. A. L. Pijnenburg ${ }^{5}$. L. Donker Kaat ${ }^{1,6}$. F. J. de Jong ${ }^{1}$ - J. C. van Swieten ${ }^{1}$. J. M. Papma ${ }^{1}$. \\ E. van den Berg ${ }^{1}$
}

Published online: 26 March 2020

(c) The Author(s) 2020

\section{Correction to: Journal of Neurology https://doi.org/10.1007/s00415-020-09738-y}

The original version of this article unfortunately contained a mistake. The presentation of Table 1 was incorrect.
The original article has been corrected. The corrected Table 1 is given below.
The original article can be found online at https://doi.org/10.1007/ s00415-020-09738-y.

\section{J. C. van Swieten}

j.c.vanswieten@erasmusmc.nl

J. M. Papma

j.papma@erasmusmc.nl

E. van den Berg

e.vandenberg@erasmusmc.nl

1 Department of Neurology, Alzheimer Center, Erasmus MC University Medical Center, Dr. Molewaterplein 40, 3000 CA Rotterdam, The Netherlands

2 Department of Radiology, Leiden University Medical Center, Leiden, The Netherlands

3 Dementia Research Center, University College London, London, UK

4 Department of Psychiatry and Psychology, Maastricht University, Maastricht, The Netherlands

5 Department of Neurology, Alzheimer Center, Location VU University Medical CenterAmsterdam Neuroscience, Amsterdam University Medical Center, Amsterdam, The Netherlands

6 Department of Clinical Genetics, Erasmus MC University Medical Center, Rotterdam, The Netherlands

F. J. de Jong

f.j.dejong@erasmusmc.nl 
Table 1 Demographic features

\begin{tabular}{|c|c|c|c|c|c|c|}
\hline & $\begin{array}{l}\text { MAPT mutation } \\
\text { carriers }(n=29)\end{array}$ & $\begin{array}{l}G R N \text { mutation } \\
\text { carriers }(n=20)\end{array}$ & $\begin{array}{l}\text { C9orf72 mutation } \\
\text { carriers }(n=31)\end{array}$ & Non-carriers $(n=24)$ & $p$ Value & Group differences \\
\hline Age at baseline, $\mathrm{y}$ & $52.6 \pm 5.5$ & $60.4 \pm 7.4$ & $62.1 \pm 9.1$ & $56.1 \pm 5.7$ & $<0.01$ & $\begin{array}{l}M A P T<G R N=C 9 \text { orf } 72 \\
\mathrm{NC}<C 9 \text { orf } 72\end{array}$ \\
\hline Sex $(\%$ female $)$ & $10(34.5 \%)$ & $12(57.1 \%)$ & $13(41.9 \%)$ & $11(45.8 \%)$ & 0.6 & n.s \\
\hline $\begin{array}{l}\text { Educational level }^{\mathrm{a}} \\
(\text { median }(\mathrm{IQR}))\end{array}$ & $5(2)$ & $5(2)$ & $5(2)$ & $5(0)$ & 0.8 & n.s \\
\hline Disease duration, y & $1.4 \pm 2.0$ & $1.0 \pm 1.1$ & $3.1 \pm 2.7$ & NA & $<0.01$ & $M A P T=G R N<C 9$ orf 72 \\
\hline $\begin{array}{l}\text { Subtype } \\
\text { dis—apa-ster }\end{array}$ & $9-15-5$ & $6-14-0$ & $6-21-3$ & NA & 0.3 & n.s \\
\hline MMSE & $25.9 \pm 2.9$ & $22.5 \pm 6.3$ & $26.5 \pm 2.7$ & $29.3 \pm 0.8$ & $<0.01$ & $\begin{array}{l}G R N<M A P T<\mathrm{NC} \\
G R N<C 9 \text { orf } 72\end{array}$ \\
\hline FAB & $14.7 \pm 3.2$ & $10.0 \pm 4.7$ & $13.9 \pm 3.4$ & $16.1 \pm 1.7$ & $<0.01$ & $G R N<M A P T=\mathrm{NC}$ \\
\hline
\end{tabular}

Values indicate mean $\pm \mathrm{SD}$ or $\mathrm{n}(\%)$ unless otherwise specified

MAPT microtubule-associated protein tau, GRN progranulin, C9orf 72 chromosome 9 open reading frame $72, N C$ non-carriers, dis disinhibited, apa apathetic, ster stereotypic, MMSE Mini-Mental State Examination, FAB Frontal Assessment Battery, n.s not significant

${ }^{a}$ Verhage Dutch educational system categorized into levels from $1=$ less than 6 years of primary education to $7=$ academic schooling

Open Access This article is licensed under a Creative Commons Attribution 4.0 International License, which permits use, sharing, adaptation, distribution and reproduction in any medium or format, as long as you give appropriate credit to the original author(s) and the source, provide a link to the Creative Commons licence, and indicate if changes were made. The images or other third party material in this article are included in the article's Creative Commons licence, unless indicated otherwise in a credit line to the material. If material is not included in the article's Creative Commons licence and your intended use is not permitted by statutory regulation or exceeds the permitted use, you will need to obtain permission directly from the copyright holder. To view a copy of this licence, visit http://creativecommons.org/licenses/by/4.0/. 